\title{
Rosa canina L. Fruit Extracts Inhibit the Growth of Bacterial Triggers of some Autoimmune Inflammatory Diseases and Potentiate the activity of Conventional Antibiotics
}

\author{
Yujun Wang ${ }^{1}$, Yushu Liang', lan Edwin Cock ${ }^{1,2, *}$ \\ 'School of Natural Sciences, Nathan Campus, Griffith University, Brisbane, AUSTRALIA. \\ 'Environmental Futures Research Institute, Nathan Campus, Griffith University, Brisbane, AUSTRALIA.
}

\begin{abstract}
Introduction: An increase in antibiotic resistance and a corresponding decrease in antimicrobial discovery have directed researchers towards alternative therapies, including plant based medicines. However, synergistic combinations of plant extracts with conventional antibiotics are a far more effective approach in overcoming resistance and potentiating the activity of antibiotics that are otherwise ineffective against resistant bacterial strains. Methods: The antibacterial activity of Rosa canina (Rosehip) extracts was investigated by disc diffusion and quantified by liquid dilution and solid phase MIC assays. The extracts were also combined with a range of conventional antibiotics and tested against various microbial triggers of autoimmune diseases. The $\Sigma F I C$ values obtained from these assays were used to determine the class of combinational effects and isobologram analysis was used to determine the ideal synergistic ratio(s). Toxicity was evaluated by Artemia nauplii and HDF cell line viability. Results: The methanolic, water and ethyl acetate extracts showed good inhibitory activity against several microbes. However, combinations of the methanolic or aqueous extracts with conventional antibiotics proved significantly more effective in inhibiting the growth of Proteus vulgaris, Klebsiella pneumonia and Acinetobacter baylyi (bacterial triggers of rheumatoid arthritis, ankylosing spondylitis and multiple sclerosis respectively). In total, 4 synergistic interactions were noted. Notably, the methanolic extract restored significant
\end{abstract}

growth inhibitory activity to chloramphenicol and tetracycline when tested in combination, thereby restoring their activity. Conclusion: Although the mechanisms of synergy are still unclear, studies indicate that compounds within $R$. canina may mimic the actions of resistance modifying agents, thus potentiating the activity of two antibiotics that are relatively ineffective alone. Isolation of these agents may be beneficial in drug design against several bacteria including the microbial triggers of rheumatoid arthritis, ankylosing spondylitis and multiple sclerosis.

Key words: Synergy, Conventional antimicrobials, Interaction, Medicinal plants, Rheumatoid arthritis, Ankylosing spondylitis, Multiple sclerosis, Drug combinations.

\section{Correspondence:}

Dr. Ian Edwin Cock

${ }^{1}$ School of Environment and Science, Nathan Campus, Griffith University, 170 Kessels Rd, Nathan, Brisbane, Queensland 4111, AUSTRALIA.

${ }^{2}$ Environmental Futures Research Institute, Griffith University, 170 Kessels Rd, Nathan, Brisbane, Queensland 4111, AUSTRALIA

Phone no: +61 737357637

E-mail: i.cock@griffith.edu.au

DOI: $10.5530 /$ pc.2020.1.3

\section{INTRODUCTION}

The improper and misuse of antibiotics has resulted in antibiotic resistance in a wide range of bacterial pathogens. ${ }^{1}$ Furthermore, the discovery of new antimicrobial agents has decreased to no more than a few new antibiotics introduced to clinical use in the last decade. ${ }^{1}$ Together, these factors have resulted in increased numbers of multi-resistant bacterial pathogens that are increasingly difficult to manage with the current range of antibiotic chemotherapies. ${ }^{2}$ The development of alternative treatment methods is crucial and considered by the World Health Organisation (WHO) to be perhaps the biggest challenge currently facing medical science. ${ }^{3}$ For a number of reasons reviewed elsewhere, ${ }^{4}$ it is unlikely that the current methods of antibiotic discovery/development will be as successful in the future.

This is especially true for the development of new effective therapies to treat autoimmune inflammatory diseases. These are a group of debilitating diseases including rheumatoid arthritis (RA), ankylosing spondylitis (AS), lupus, Lyme disease, multiple sclerosis (MS), celiac disease and rheumatic fever (RV). ${ }^{5}$ All of these diseases result from an abnormal immune response to self-tissue as a consequence of antigen challenge, often by bacterial pathogens. There is currently no cure for any of these diseases and the current treatment strategy is to alleviate the symptoms with analgesics and anti-inflammatory therapies. However, as RA, AS, MS and $\mathrm{RV}$ are induced in genetically people by bacterial pathogens, a more effective preventative treatment may be to target the growth of the specific trigger bacteria, thereby blocking the disease etiological events. ${ }^{5}$ Whilst antibiotics are already available for the treatment of all of these bacteria, the development of resistant strains in recent years have decreased their efficacy towards some strains. ${ }^{1}$ Furthermore, the prophylactic use of pure antibiotics over a long term would certainly induce resistance, thereby rendering the bacteria refractory to their actions. A better approach may be to use combinations of antibacterial components. ${ }^{4}$

Traditional medicines have great potential for antimicrobial drug development. Despite this, relatively few plant derived antibiotic compounds are in common use clinically. This may be because synergistic interactions are often required to potentiate the antibacterial activity and purified compounds often have much lower activity than the crude extracts. ${ }^{6}$ A combinational approach that allows synergistic interaction between plant extracts (or pure plant compounds) and conventional antibiotics may be more effective in combatting bacterial pathogens, especially antibiotic resistant strains ${ }^{7,8}$ Combinational therapy is already preferred over mono-therapy to treat multiple life-threatening infectious diseases such as malaria, tuberculosis and HIV/AIDS due to its ability to target multiple facets of a disease and to curb resistance. ${ }^{4}$ A combination of plant extracts/isolated compounds with conventional antibiotics may also prove to have an economic advantage. ${ }^{6}$ Developing a new drug requires years of extensive and costly testing. However, combinational therapy can potentially restore an existing drug to a state of significantly reduced resistance, thereby bypassing the strenuous and expensive process of discovering new antimicrobial agents. ${ }^{6}$ Further advantages of synergistic interactions include increased efficiency, reduced side effects, increased stability and bioavailability and the requirement for lower doses in comparison to synthetic alternatives. ${ }^{6}$ 
Rosa canina L. (commonly known as dog rose) is wild rose species native to Europe, northwest Africa and western Asia. Following flowering, bright red fruit known as rose hips form. The rose hips are used for the production of jams, jellies, beverages and soups. They are also consumed as herbal infusions and may be eaten raw. Rose hips are high in ascorbic acid. Indeed, the fresh fruit may contain up to $1.5 \%$ ascorbic acid (by mass). ${ }^{9}$ Relatively high levels of $\beta$-carotene, lutein, zeaxanthin and lycopene have also been detected in rose hips. ${ }^{10,11}$ Many of these compounds have beneficial therapeutic properties in the treatment of arthritis, gout, urinary tract infections, fever, cold and as a diuretic and laxative. ${ }^{11}$ Despite the interesting phytochemistry and its therapeutic properties, $R$. canina remains poorly investigated. Notably, a hydroalcoholic $R$. canina rose hip extract was recently reported to have good anti-inflammatory activity. Despite this, $R$. canina extracts are yet to be tested against the bacterial triggers of rheumatoid arthritis (Proteus mirabilis), ankylosing spondylitis (Klebsiella pneumoniae), multiple sclerosis (Acinetobacter baylyi, Pseudomonas aeruginosa) and rheumatic fever (Streptococcus pyogenes). ${ }^{5}$ Furthermore, we were unable to find any studies testing the antibacterial activity of $R$. canina extracts in combination with conventional antibiotics. Therefore, this study was undertaken to investigate the antimicrobial effects of $R$. canina rose hip extracts and their ability to potentiate the growth inhibitory properties of conventional antibiotics against the bacterial triggers of some autoimmune inflammatory diseases.

\section{MATERIALS AND METHODS}

\section{Plant source and extraction}

Certified Rosa canina L. fruit powder was obtained from Noodles Herbal Emporium, Australia and a voucher specimen (GU2017RP) was deposited in the School of Natural Sciences, Griffith University, Australia. Individual $1 \mathrm{~g}$ masses of the ground rosehip material were weighed into separate $50 \mathrm{~mL}$ Falcon tubes and $50 \mathrm{~mL}$ of methanol, deionised water, ethyl acetate, chloroform or hexane were individually added. All solvents were obtained from Ajax Fine Chemicals, Australia and were AR grade. The ground plant materials were extracted in each solvent for $24 \mathrm{~h}$ at $4^{\circ} \mathrm{C}$ with gentle shaking. The extracts were filtered through Whatman No. 54 filter paper under vacuum. The solvent extracts were air dried at room temperature in the shade. The aqueous extracts were lyophilised by freeze drying at $-50^{\circ} \mathrm{C}$. The resultant dried extracts were weighed to determine the extraction yield and then dissolved in $10 \mathrm{~mL}$ deionised water (containing 1\% DMSO).

\section{Qualitative phytochemical studies}

Phytochemical analysis of the R. canina extracts for the presence of alkaloids, cardiac glycosides, flavonoids, phenolic compounds, phytosterols, saponins, tannins and triterpenoids was achieved as previously described. ${ }^{12,13}$

\section{Antibacterial screening Conventional Antibiotics}

Penicillin-G (1440-1680 $\mu \mathrm{g} / \mathrm{mg}$ ), chloramphenicol ( $\geq 98 \%$ purity), erythromycin $(\geq 850 \mu \mathrm{g} / \mathrm{mg})$, gentamycin $(600 \mu \mathrm{g} / \mathrm{mg})$ and tetracycline $(\geq 95 \%$ purity) were purchased from Sigma-Aldrich, Australia and used for the microplate liquid dilution assay. All antibiotics were prepared in sterile deionised water at stock concentrations of $0.01 \mathrm{mg} / \mathrm{mL}$ and stored at $4{ }^{\circ} \mathrm{C}$ until use. For the disc diffusion studies, ampicillin $(10 \mu \mathrm{g})$ and chloramphenicol discs $(10 \mu \mathrm{g})$ standard discs were obtained from Oxoid Ltd., Australia and used as positive controls.

\section{Bacterial cultures}

All bacterial strains were selected based on their ability to trigger autoimmune inflammatory diseases in genetically susceptible individuals. ${ }^{5}$ Reference strains of Proteus mirabilis (ATCC21721), Proteus vulgaris (ATCC21719), Klebsiella pneumoniae (ATCC31488), Acinetobacter baylyi (ATCC33304) and Pseudomonas aeruginosa (ATCC39324) were purchased from American Type Culture Collection, USA. A clinical isolate strain of Streptococcus pyogenes was obtained from the School of Environment and Science teaching laboratory, Griffith University, Australia. All bacteria were cultured in nutrient broth (Oxoid Ltd., Australia). Streak nutrient agar (Oxoid Ltd., Australia) plates were tested in parallel to ensure the purity of all bacterial cultures and for sub-culturing. All bacterial cultures were incubated at $37^{\circ} \mathrm{C}$ for $24 \mathrm{~h}$ and were subcultured and maintained in nutrient broth at $4^{\circ} \mathrm{C}$ until use.

\section{Evaluation of antibacterial activity}

Antibacterial activity screening of the $R$. canina extracts was assessed using a modified disc diffusion assay. ${ }^{12,13}$ Ampicillin (10 $\mu$ g) and chloramphenicol discs $(10 \mu \mathrm{g})$ were obtained from Oxoid Ltd., Australia and used as positive controls to compare antibacterial activity. Filter discs infused with $10 \mu \mathrm{L}$ of distilled water (containing $1 \%$ DMSO) were used as a negative control.

\section{Minimum inhibitory concentration (MIC) determination}

The minimum inhibitory concentration for each extract was determined using two methods. A liquid dilution MIC assay was employed as it is generally considered the most sensitive bacterial growth inhibitory assay. ${ }^{14}$ Furthermore, as microplate liquid dilution MIC assays are perhaps the most commonly used method of quantifying bacterial growth inhibition efficacy, use of this method allows for comparisons with other studies. A solid phase agar disc diffusion assay was also used in this study for comparison.

\section{Microplate liquid dilution MIC assay}

The MICs of the extracts were evaluated by standard methods. ${ }^{15}$ All plates were incubated at $37^{\circ} \mathrm{C}$ for $24 \mathrm{~h}$. p-Iodonitrotetrazolium violet (INT) was obtained from Sigma-Aldrich, Australia and dissolved in sterile deionised water to prepare a $0.2 \mathrm{mg} / \mathrm{mL}$ INT solution. A $40 \mu \mathrm{L}$ volume of this solution was added into all wells and the plates were incubated for a further $6 \mathrm{~h}$ at $37^{\circ} \mathrm{C}$. Following incubation, the MIC was visually determined as the lowest dose at which colour development was inhibited.

\section{Disc diffusion MIC assay}

The minimum inhibitory concentrations (MIC) of the extracts was also evaluated by disc diffusion assay as previously described. ${ }^{12,13}$ Graphs of the zone of inhibition versus ln concentration were plotted and MIC values were achieved using linear regression.

\section{Sum of fractional inhibitory concentration $(\Sigma \mathrm{FIC})$ assessment}

Interactions between the $R$. canina extracts and the conventional antibiotics were examined by determination of the sum of fractional inhibitory concentrations ( $\Sigma$ FIC) for each combination. ${ }^{15}$ The FIC values for each component ( $\mathrm{a}$ and $\mathrm{b}$ ) were calculated using the following equations where a represents the plant extract sample and b represents the conventional antibiotic:

$$
\text { FIC }(\mathrm{a})=\left(\frac{\text { MIC }[\mathrm{a} \text { in combination with } \mathrm{b}]}{\text { MIC }[\mathrm{a} \text { independently }]}\right)
$$




$$
\mathrm{FIC}(\mathrm{b})=\left(\frac{\mathrm{MIC}[\mathrm{b} \text { in combination with } \mathrm{a}]}{\mathrm{MIC}[\mathrm{b} \text { independently }]}\right)
$$

The $\Sigma$ FIC was then calculated using the formula $\Sigma$ FIC $=$ FIC $(a)+F I C(b)$. The interactions were classified as synergistic $(\Sigma \mathrm{FIC} \leq 0.5)$, additive ( $\Sigma$ FIC $>0.5-1.0)$, indifferent $(\Sigma F I C>1.0-4.0)$ or antagonistic $\left(\sum\right.$ FIC $\left.>4.0\right) .{ }^{15}$

\section{Varied ratio combination studies (isobolograms)}

For each combination producing synergistic interactions, nine different ratios spanning the range 10:90 (extract:antibiotic) to 90:10 (extract:antibiotic) were tested. All combinations were tested in duplicate in two independent experiments, providing four replicates for each combination ratio. The data is presented as the mean of four replicates. Data points for each ratio examined were plotted on a isobologram and this was used to determine optimal combination ratios to obtain synergy. Data points on or below the 0.5:0.5 line indicate synergy; those above the 0.5:0.5 line, up to and including the 1.0:1.0 line indicate an additive interaction; data points above the 1.0:1.0 line indicate indifferent interaction.

\section{Toxicity screening}

Two assays were used to assess the toxicity of the individual samples. The Artemia nauplii lethality assay (ALA) was utilised for rapid preliminary toxicity screening, whereas the MTT cellular proliferation assay was used to determine a cellular evaluation of toxicity.

\section{Artemia franciscana Kellogg nauplii toxicity screening}

Potassium dichromate $\left(\mathrm{K}_{2} \mathrm{Cr}_{2} \mathrm{O}_{7}\right)$ (AR grade, Chem-Supply, Australia) was prepared in deionised water $(4 \mathrm{mg} / \mathrm{mL})$ and serially diluted in artificial seawater as a reference toxin. Toxicity of the $R$. canina extracts, reference toxin and conventional antibiotics was assessed using a modified Artemia franciscana nauplii lethality assay. ${ }^{16} \mathrm{The}^{\mathrm{LC}} \mathrm{C}_{50}$ with $95 \%$ confidence limits for each treatment was calculated using probit analysis.

\section{Cellular viability assay}

All $R$. canina extracts were also screened individually using a normal human primary dermal fibroblast (HDF) standard assay. ${ }^{17}$ Briefly, the HDF cells were obtained from American Type Culture Collection (ATCC PCS-201-012) and were cultured and maintained in Dulbecco's modified eagle medium (DMEM; Thermo Fisher Scientific, Australia), supplemented with $10 \%$ foetal calf serum (Life Technologies, Australia), $50 \mu \mathrm{g} / \mathrm{mL}$ streptomycin (Sigma-Aldrich, Australia) and $50 \mathrm{IU} / \mathrm{mL}$ penicillin (Sigm-Aldricha, Australia) at $37^{\circ} \mathrm{C}, 5 \% \mathrm{CO}_{2}$ in a humidified atmosphere. Individual $70 \mu \mathrm{L}$ volumes of culture media (containing approximately 5000 cells) were added to wells of a 96 well plate and $30 \mu \mathrm{L}$ of the test extracts or cell media (for the negative control) was added to each well. The plates were incubated at $37^{\circ} \mathrm{C}, 5 \% \mathrm{CO}_{2}$ for $24 \mathrm{~h}$ in a humidified atmosphere. All extracts were screened at $200 \mu \mathrm{g} / \mathrm{mL}$. The cells were then washed in PBS ( $\mathrm{pH}$ 7.2) to remove interference due to sample colour. A $20 \mu \mathrm{L}$ volume of Cell Titre 96 Aqueous One solution (Promega) was added to each well and the plates were incubated for a further $3 \mathrm{~h}$. Absorbances were recorded at a test wavelength of 540nm and a blank wavelength of 690nm using a Molecular Devices, Spectra Max M3 plate reader. All tests were performed in at least triplicate and triplicate controls were included on each plate. The \% cellular viability of each test was calculated using the following formula:

$\%$ cellular viability $=\frac{\text { Abs test sample }- \text { (mean Abs control }- \text { mean Abs blank })}{(\text { mean Abs control }- \text { mean Abs blank })}$

Cellular viability $\leq 50 \%$ of the untreated control indicated toxicity, whereas extracts or controls with $>50 \%$ untreated control viability were deemed to be nontoxic.

\section{Statistical analysis}

Data is expressed as the mean \pm SEM of at least three independent experiments. One-way ANOVA was used to calculate statistical significance between the negative control and treated groups with a $P$ value $<0.01$ considered to be statistically significant.

\section{RESULTS}

Liquid extraction yields ranged from $38 \mathrm{mg}$ ( $R$. canina hexane extract) to $312 \mathrm{mg}$ (aqueous $R$. canina extract) (Table 1). Qualitative phytochemical screening (Table 1) showed that the higher polarity solvents (methanol and water) extracted the greatest mass and widest diversity of phytochemical classes.

\section{Bacterial growth inhibition screening}

Inhibition of bacterial triggers of rheumatoid arthritis (P. mirabilis and $P$. vulgaris)

P. mirabilis growth was inhibited by the mid to high polarity $R$. canina water, methanol and ethyl acetate extracts (Figure 1). The methanolic extract was the strongest $P$. mirabilis growth (as judged by ZOI), with a ZOI of $8.6 \pm 0.4 \mathrm{~mm}$. A volume of $10 \mu \mathrm{L}$ of this extract was infused into the disc, which equates to approximately $100 \mu \mathrm{g}$ of extract infused into the disc. The ZOI for this extract is substantially smaller than that of the ampicillin $(13.3 \pm 0.6 \mathrm{~mm})$ and chloramphenicol controls $(12.5 \pm 0.5 \mathrm{~mm})$. However, it is noteworthy that these control antibiotics were tested pure and at relatively high doses $(10 \mu \mathrm{g} /$ disc $)$. In contrast, the extracts were crude mixtures and the antimicrobial compounds would be expected to account for a small \% of the total extract mass. Therefore, the methanolic extract is an effective inhibitor of $P$. mirabilis growth and may be effective in the prevention and treatment of rheumatoid arthritis. The aqueous and ethyl acetate extracts had similar ZOIs to the methanolic extract. In contrast, the chloroform and hexane extracts were completely ineffective against $P$. mirabilis growth. Similar inhibitory trends were noted for $P$. vulgaris growth (Figure 2), although slightly smaller ZOIs were measured. As for $P$. mirabilis, the methanolic extract was the strongest

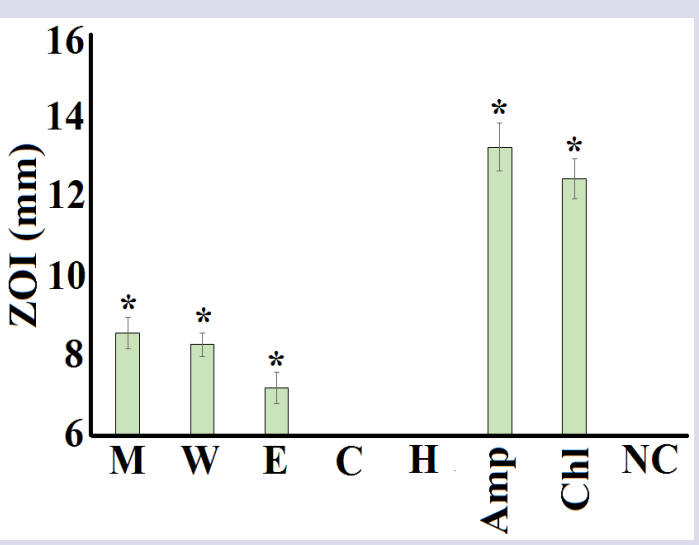

Figure 1: Antibacterial activity of R. canina extracts against $P$. mirabilis (ATCC21721) measured as zones of inhibition $(\mathrm{mm}) . \mathrm{M}=$ methanolic extract; $\mathrm{W}=$ aqueous extract; $\mathrm{E}=$ ethyl acetate extract; $\mathrm{C}=$ chloroform extract; $\mathrm{H}=$ hexane extract. The positive controls were Amp (ampicillin $10 \mu \mathrm{g})$ and $\mathrm{Chl}(\mathrm{chloramphenicol} 10 \mu \mathrm{g})$. Negative control $(\mathrm{NC})=$ water. Results are expressed as mean zones of inhibition of two repeats, each with internal triplicates $(n=6) \pm$ SEM. ${ }^{*}$ indicates results that are significantly different to the negative control $(P<0.01)$. 
inhibitor of $P$. vulgaris growth $(\mathrm{ZOI}=7.7 \pm 0.3 \mathrm{~mm})$. The aqueous and ethyl acetate extracts had similar efficacy, with ZOIs of $7.3 \pm 0.3 \mathrm{~mm}$ and $6.7 \pm 0.3 \mathrm{~mm}$ respectively. In contrast, larger ZOIs were measured for the ampicillin $(10.0 \pm 0.5 \mathrm{~mm})$ and chloramphenicol controls $(11.3 \pm 0.6 \mathrm{~mm})$. All other extracts were ineffective at inhibiting $P$. vulgaris growth.

\section{Inhibition of a bacterial trigger of ankylosing spondylitis ( $K$. pneumoniae)}

Only the methanol and hexane $R$. canina extracts inhibited the growth of $K$. pneumoniae (Figure 3). The methanolic extract was the strongest growth inhibitor, albeit with a relatively small ZOI $(7.5 \pm 0.5 \mathrm{~mm})$ compared with that seen for the Proteus spp. The hexane extracts also inhibited $K$. pneumoniae growth, although the ZOI $(6.7 \pm 0.3 \mathrm{~mm})$ is indicative of only weak antibacterial activity. As K. pneumoniae can induce ankylosing spondylitis in genetically susceptible individuals, ${ }^{5}$ these extracts may be beneficial in the prevention and treatment of that disease. In contrast, this bacterium was highly susceptible to the ampicillin and chloramphenicol controls, with inhibition zones of $11.7 \pm 0.6 \mathrm{~mm}$ and $9.8 \pm 0.4 \mathrm{~mm}$ respectively. No growth inhibition was detected for the mid to lower polarity chloroform and ethyl acetate extracts.

\section{Inhibition of bacterial triggers of multiple sclerosis (A. baylyi and $P$. aeruginosa)}

Only the methanolic $R$. canina extract inhibited A. baylyi growth, albeit with a small ZOI $(6.8 \pm 0.3 \mathrm{~mm})$, indicative of weak inhibitory activity (Figure 4). However, it is noteworthy that this bacterium was also relatively resistant to ampicillin $(\mathrm{ZOI}=6.8 \pm 0.3 \mathrm{~mm})$. In contrast, this $A$. baylyi strain was highly susceptible to chloramphenicol (ZOI $=11.3 \pm 0.6 \mathrm{~mm})$. Similarly, only the methanolic extract inhibited $P$. aeruginosa growth (Figure 5). However, the $6.6 \pm 0.3 \mathrm{~mm}$ ZOI measured for this extract indicated only weak growth inhibitory activity. However, the P. aeruginosa strain tested in this study was resistant to both the ampicillin and chloramphenicol controls, each inducing zones of inhibition of only approximately $6.5 \mathrm{~mm}$. Thus, the methanolic $R$. canina extract may still be useful in prevention and treating $P$. aeruginosa infections. As A. baylyi and $P$. aeruginosa can both induce multiple sclerosis in genetically susceptible people, ${ }^{5}$ the methanolic $R$. canina extract may be useful in the prevention and treatment of that disease, as well as other illnesses caused by these

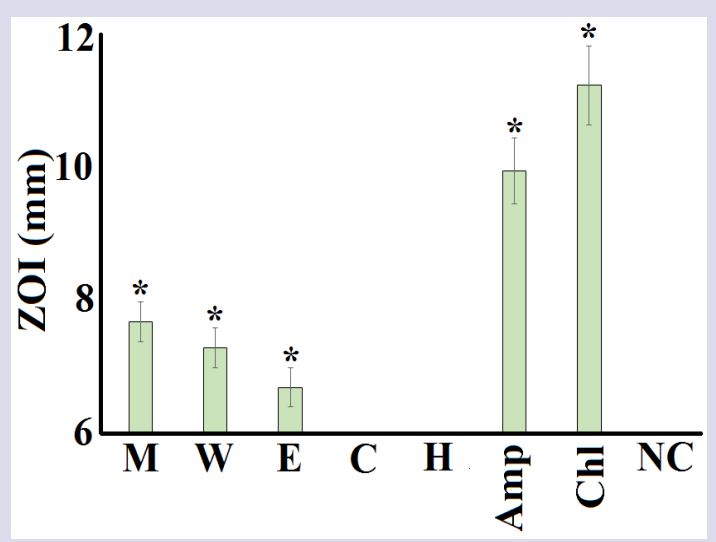

Figure 2: Antibacterial activity of $R$. canina extracts against $P$. vulgaris (ATCC21719) measured as zones of inhibition $(\mathrm{mm}) . \mathrm{M}=$ methanolic extract; $W=$ aqueous extract; $E=$ ethyl acetate extract; $C=$ chloroform extract; $\mathrm{H}=$ hexane extract. The positive controls were Amp (ampicillin $10 \mu \mathrm{g}$ ) and $\mathrm{Chl}$ (chloramphenicol 10 $\mathrm{gg}$ ). Negative control $(\mathrm{NC})=$ water. Results are expressed as mean zones of inhibition of two repeats, each with internal triplicates $(n=6) \pm$ SEM. ${ }^{*}$ indicates results that are significantly different to the negative control $(P<0.01)$. bacteria.

Inhibition of the bacterial trigger of rheumatic fever (S. pyogenes)

$S$. pyogenes growth was inhibited by the methanolic and aqueous $R$. canina extracts (Figure 6). All other extracts were completely ineffective at inhibiting the growth of this bacterium. The methanolic extract was particularly potent, with a ZOI of $9.3 \pm 0.6 \mathrm{~mm}$. Indeed, the methanolic extract had stronger relative activity than the ampicillin $(8.2 \pm 0.4 \mathrm{~mm})$ and chloramphenicol controls $(6.8 \pm 0.3 \mathrm{~mm})$. This is particularly noteworthy as both positive controls are pure compounds and were tested at relatively high doses $(10 \mu \mathrm{g} / \mathrm{disc})$. The water extract also inhibited $S$. pyogenes growth, albeit with substantially smaller ZOIs indicative of only low to moderate growth inhibitory activity. As $S$. pyogenes can trigger rheumatic fever in genetically susceptible people, ${ }^{5}$ the $R$. canina methanolic extract (and to a lesser extent, the aqueous extract) may be effective in the prevention and treatment of this disease (and other diseases caused by this bacterium).

\section{Quantification of Minimum Inhibitory Concentration (MIC)}

The relative antimicrobial strength of the extracts was further evaluated by determining the MIC values using two methods: the liquid dilution MIC assay and the disc diffusion MIC assay (Table 2). Consistent with the antibacterial screening assays, each of the higher polarity methanol and water $R$. canina extracts were generally effective at inhibiting the growth of all of the bacteria tested. The lack of inhibition of $K$. pneumonia by the aqueous extract was the only exception to this trend. The MIC values of the conventional antibiotic controls were only determined for the liquid dilution assay. Commercially manufactured discs with set amounts of antibiotics loaded were used for the disc diffusion assay and thus the zones of only single doses were recorded. Gentamycin was the most potent antibiotic (as judged by its MIC) and inhibited the widest range of bacterial species. Indeed, all bacterial species tested were susceptible to this antibiotic. Notably, the $P$. aeruginosa strain used in these studies was resistant to all of antibiotics except gentamycin. Furthermore, with the exception of $P$. mirabilis and $P$. vulgaris, all of the other bacterial strains were completely resistant to penicillin.

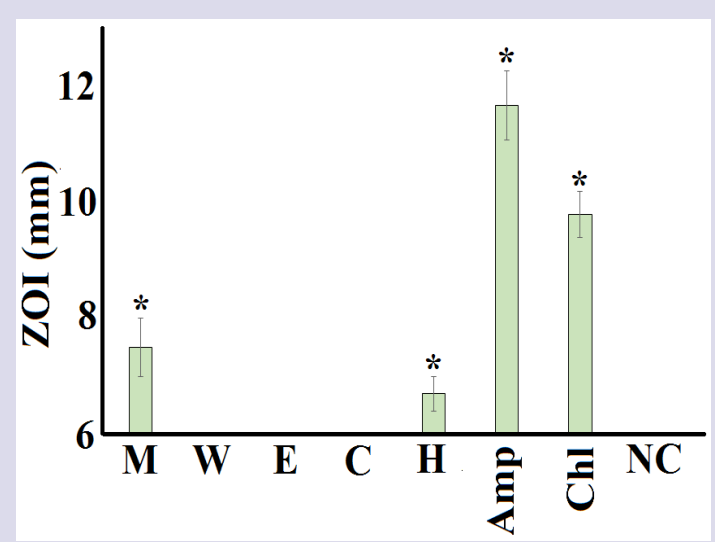

Figure 3: Antibacterial activity of $R$. canina extracts against extracts against K. pneumoniae (ATCC31488) measured as zones of inhibition (mm). $M=$ methanolic extract; $W=$ aqueous extract; $E=$ ethyl acetate extract; $\mathrm{C}=$ chloroform extract; $\mathrm{H}=$ hexane extract. The positive controls

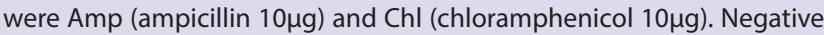
control $(\mathrm{NC})=$ water. Results are expressed as mean zones of inhibition of two repeats, each with internal triplicates $(n=6) \pm$ SEM. * indicates results that are significantly different to the negative control $(P<0.01)$. 
The MIC values determined for the $R$. canina extracts compare relatively well between the disc diffusion and liquid dilution assays with few exceptions. All bacterial species were generally most susceptible to the methanolic extract, although the ethyl acetate extract had similar efficacy towards the Proteus spp. (based on MIC values). The growth of $P$. mirabilis was inhibited by methanolic (DD MIC $200 \mu \mathrm{g} / \mathrm{mL}$; LD MIC $150 \mu \mathrm{g} / \mathrm{mL}$ ) and ethyl acetate extracts (DD MIC $200 \mu \mathrm{g} / \mathrm{mL}$; LD MIC $185 \mu \mathrm{g} / \mathrm{mL}$ ) with MIC values that indicate strong growth inhibitory activity. The aqueous

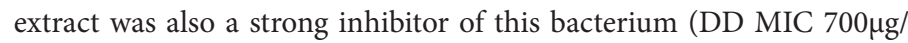
$\mathrm{mL}$; LD MIC $525 \mu \mathrm{g} / \mathrm{mL}$ ). Similar, albeit slightly higher MIC values were also determined for these extracts against $P$. vulgaris. Therefore, these extracts may be useful in the prevention and treatment of rheumatoid arthritis. The methanolic extract was also a good inhibitor of K. pneu-

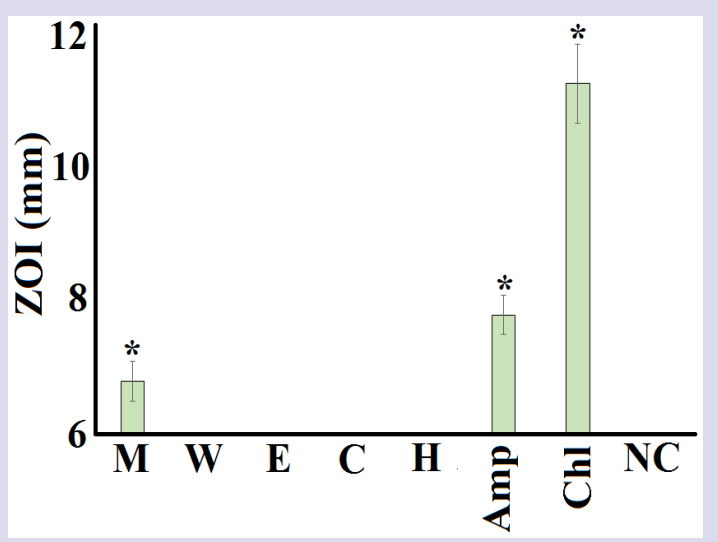

Figure 4: Antibacterial activity of $R$. canina extracts against $A$. baylyi (ATCC33304) measured as zones of inhibition $(\mathrm{mm}) . M=$ methanolic extract; $\mathrm{W}=$ aqueous extract; $\mathrm{E}=$ ethyl acetate extract; $\mathrm{C}=$ chloroform extract; $\mathrm{H}=$ hexane extract. The positive controls were Amp (ampicillin $10 \mu \mathrm{g}$ ) and $\mathrm{Chl}$ (chloramphenicol 10 $\mu \mathrm{g}$ ). Negative control $(\mathrm{NC})=$ water. Results are expressed as mean zones of inhibition of two repeats, each with internal triplicates $(n=6) \pm$ SEM. * indicates results that are significantly different to the negative control $(P<0.01)$.

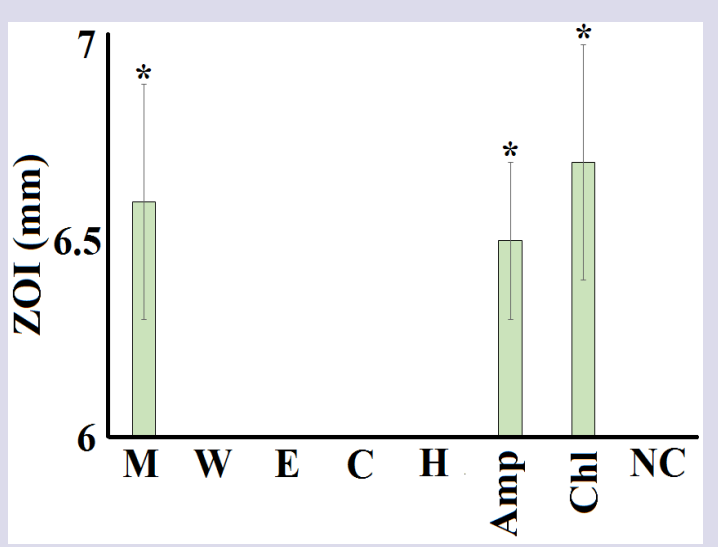

Figure 5: Antibacterial activity of $R$. canina extracts against $P$. aeruginosa (ATCC39324) measured as zones of inhibition ( $\mathrm{mm}$ ). $\mathrm{M}=$ methanolic extract; $W=$ aqueous extract; $E$ = ethyl acetate extract; $C=$ chloroform extract; $\mathrm{H}=$ hexane extract. The positive controls were Amp (ampicillin $10 \mu \mathrm{g})$ and $\mathrm{Chl}(\mathrm{chloramphenicol} 10 \mu \mathrm{g})$. Negative control (NC) $=$ water. Results are expressed as mean zones of inhibition of two repeats, each with internal triplicates $(n=6) \pm$ SEM. * indicates results that are significantly different to the negative control $(P<0.01)$. moniae growth (DD MIC $870 \mu \mathrm{g} / \mathrm{mL}$; LD MIC $650 \mu \mathrm{g} / \mathrm{mL}$ ). Similarly, the hexane extract was a potent inhibitor of this bacterium (DD MIC $164 \mu \mathrm{g} / \mathrm{mL}$; LD MIC $82 \mu \mathrm{g} / \mathrm{mL}$ ) and thus may also be useful in the prevention and treatment of ankylosing spondylitis. Whilst the methanolic and aqueous also inhibited the growth of $A$. baylyi, P. aeruginosa and $S$. pyogenes, the MIC values were generally $>1000 \mu \mathrm{g} / \mathrm{mL}$, indicating only low to moderate potency.

\section{Fractional inhibitory concentration (FIC) assessment}

\section{Combinational effects on a bacterial trigger of rheumatoid arthritis} (Proteus spp.)

A range of interactions was evident for combinations of the $R$. canina extracts with conventional antibiotics when tested against $P$. mirabilis and $P$. vulgaris (Table 3). $\Sigma$ FIC values could not be determined for the majority of the combinations as one or both of the components in the combination were in effective against the tested bacterium. Of the effective combinations, the majority of were non-interactive (approximately $79 \%$ of the inhibitory combinations). Whilst these combinations have no additional benefit over the individual monotherapies alone, the lack of antagonism indicates that taking these therapies in combination would not have detrimental effects. This is important information as often allopathic and complementary therapies may be taken concurrently. Notably, the methanolic extract induced synergistic effects in combination with tetracycline $(\Sigma \mathrm{FIC}=0.37)$. This combination may therefore be useful in the prevention and treatment of rheumatoid arthritis. The aqueous and ethyl acetate extracts also potentiated the activity of tetracycline, albeit to a lesser extent than the methanol extract, producing additive effects. Therefore, these combinations may also be useful in preventing and treating rheumatoid arthritis, as well as any other disease caused by Proteus spp. infections (e.g. urinary tract infections).

Combinational effects on a bacterial trigger of ankylosing spondylitis ( $K$. pneumoniae)

Two synergistic interactions were noted for combinations of the R. cani$n a$ extracts and conventional antibiotics against the growth of K. pneumonia (Table 3). Interestingly, both of these combinations contained the methanolic extract and either chloramphenicol or tetracycline $(\Sigma \mathrm{FIC}$



Figure 6: Antibacterial activity of $R$. canina extracts against a clinical isolate of $S$. pyogenes measured as zones of inhibition $(\mathrm{mm}) . \mathrm{M}=$ methanolic extract; $W=$ aqueous extract; $E=$ ethyl acetate extract; $C=$ chloroform extract; $\mathrm{H}=$ hexane extract. The positive controls were Amp (ampicillin $10 \mu \mathrm{g})$ and $\mathrm{Chl}$ (chloramphenicol 10 $\mu \mathrm{g}$ ). Negative control (NC) = water. Results are expressed as mean zones of inhibition of two repeats, each with internal triplicates $(n=6) \pm$ SEM. ${ }^{*}$ indicates results that are significantly different to the negative control $(P<0.01)$. 

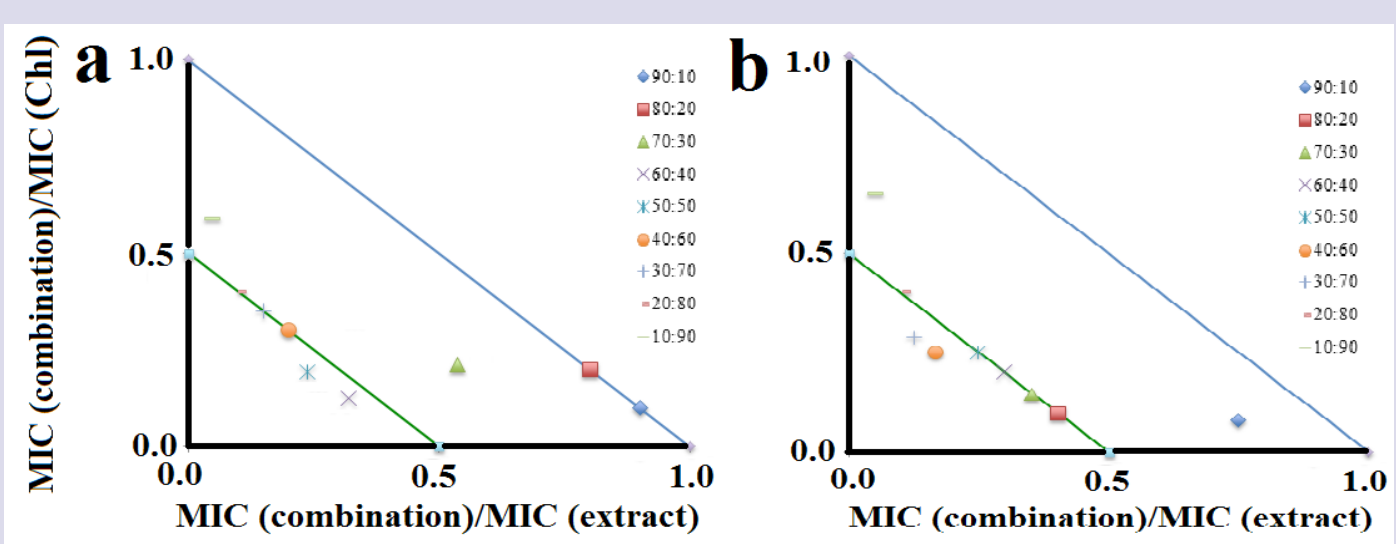

Figure 7: Isobologram for combinations of chloramphenicol and methanolic $R$. canina extract against (a) K. pneumonia and (b) A. baylyi. Results represent mean FIC values of four replicates. Ratio $=\%$ extract:\% antibiotic. Ratios lying on or underneath the 0.5:0.5 line are considered to be synergistic ( $\Sigma F I C \leq 0.5$ ). Any points between the 0.5:0.5 and 1.0:1.0 lines are deemed additive ( $\mathrm{FIC}>0.5-1.0)$. $\mathrm{Chl}=$ chloramphenicol.
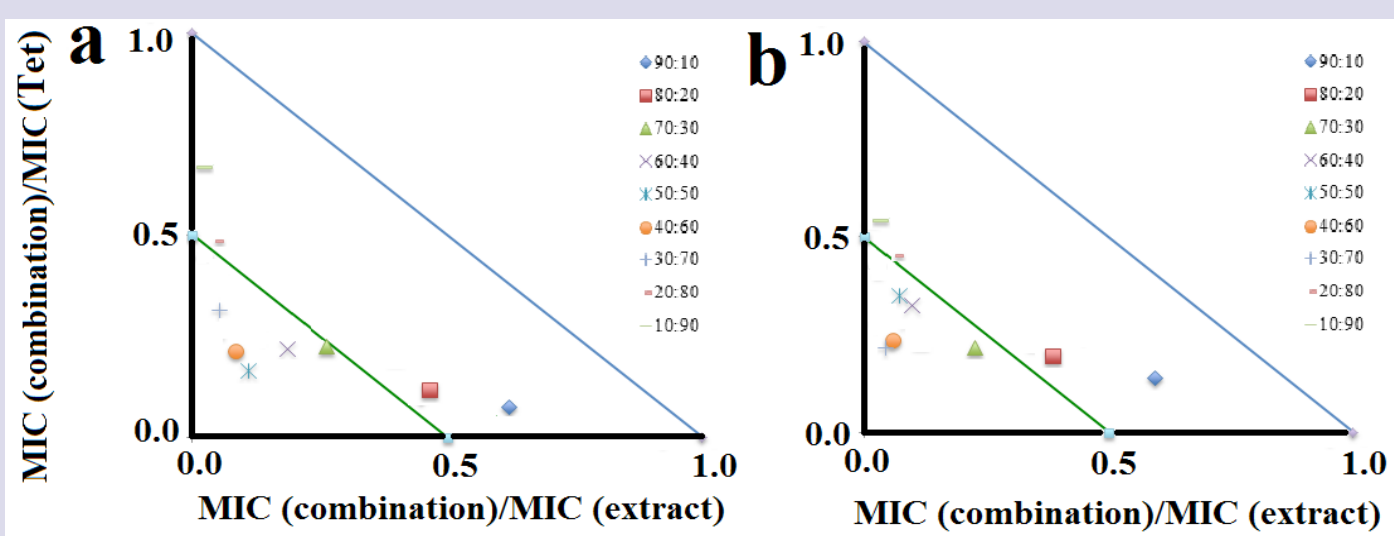

Figure 8: Isobologram for combinations of tetracycline and methanolic R. canina extract against (a) P. vulgaris and (b) $K$. pneumonia. Results represent mean FIC values of four replicates. Ratio $=\%$ extract:\% antibiotic. Ratios lying on or underneath the 0.5:0.5 line are considered to be synergistic ( $\Sigma F I C \leq 0.5)$. Any points between the 0.5:0.5 and 1.0:1.0 lines are deemed additive $(\Sigma \mathrm{FIC}>0.5-1.0)$. Tet $=$ tetracycline.

0.46 and 0.29 respectively). These combinations may therefore be effective in the prevention and treatment of ankylosing spondylitis (and other diseases caused by K. pneumonia). Combination of the same antibiotics with the hexane extract produced additive effects, indicating that these combinations may also be beneficial in the treatment of those diseases due to their increased growth inhibitory efficacies compared to the individual components. The majority of the other combinations were either non-interactive or ineffective.

\section{Combinational effects on bacterial triggers of multiple sclerosis ( $A$. baylyi and P. aeruginosa)}

A range of interactions were observed between $R$. canina extracts and conventional antibiotics against A. baylyi (Table 3). The combination of the methanolic extract with chloramphenicol resulted in a synergistic interaction $(\Sigma \mathrm{FIC}=0.5)$. A further two combinations were additive in combination with tetracycline. Thus, these combinations may be beneficial due to their increased growth inhibitory efficacies. These combinations include methanolic $(\Sigma \mathrm{FIC}=0.6)$ and aqueous $(\Sigma \mathrm{FIC}=0.8)$ extracts in combination with tetracycline. As A. baylyi is one of the bacterial triggers of multiple sclerosis, ${ }^{5}$ combinations may be beneficial in the prevention and treatment of that disease. Table 3 also summarises the interactions of the $R$. canina extracts and conventional antibiotics against $P$. aeruginosa. Most combinations were ineffective at inhibiting $P$. aeruginosa growth and therefore $\Sigma$ FIC values could not be determined. The methanolic and aqueous extracts in combination with gentamycin were non-interactive. Whilst there is no added benefit in combining these therapies, their concurrent use would not decrease the activity of either component and therefore they may be safely used in combination without decreasing the efficacy of the treatment.

\section{Combinational effects on a bacterial trigger of rheumatic fever (S. pyogenes)}

The combinational antimicrobial effects of the $R$. canina extracts with various conventional antibiotics against $S$. pyogenes are summarised in Table 3. No synergistic effects were detected for any combination. The only additive combination detected was for the methanolic extract and tetracycline $(\Sigma \mathrm{FIC}=0.8)$. As this combination produces increased efficacy compared to either monotherapy alone, it may be beneficial in treating $S$. pyogenes infections. This combination may therefore be useful in the prevention of rheumatic fever in genetically susceptible individuals, 
Table 1: The mass of dried extracted material, the concentration after resuspension in deionised water and qualitative phytochemical screenings of the R. canina extracts.

\begin{tabular}{|c|c|c|c|c|c|c|c|c|c|c|c|c|c|c|c|}
\hline 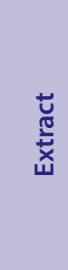 & 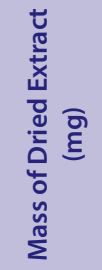 & 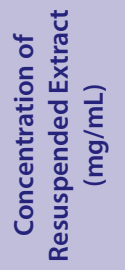 & 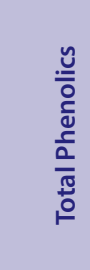 & 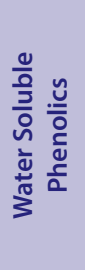 & 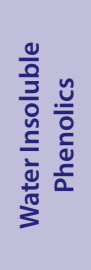 & 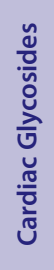 & 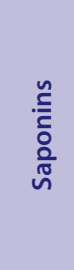 & 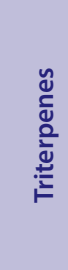 & 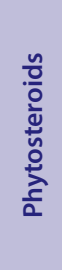 & 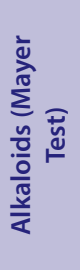 & 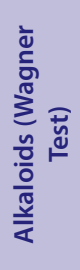 & $\begin{array}{l}\frac{n}{0} \\
\frac{0}{0} \\
\frac{c}{0} \\
\frac{\pi}{\pi} \\
\frac{\pi}{4}\end{array}$ & 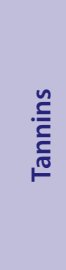 & 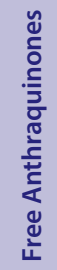 & 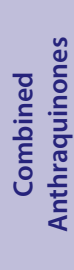 \\
\hline M & 108 & 10.8 & +++ & ++ & +++ & - & ++ & ++ & - & - & - & +++ & ++ & - & - \\
\hline W & 312 & 31.2 & +++ & ++ & ++ & - & ++ & + & - & + & + & +++ & ++ & - & - \\
\hline E & 81 & 8.1 & + & + & - & - & - & - & - & - & - & ++ & - & - & - \\
\hline C & 46 & 4.6 & + & - & + & - & + & - & - & - & - & + & - & - & - \\
\hline $\mathrm{H}$ & 38 & 3.8 & + & - & + & - & - & + & - & - & - & - & - & - & - \\
\hline
\end{tabular}

+++ indicates a large response; ++ indicates a moderate response; + indicates a minor response; - indicates no response in the assay. $\mathrm{W}=$ aqueous extract; $\mathrm{M}=$ methanolic extract; $\mathrm{C}=$ chloroform extract; $\mathrm{H}=$ hexane extract; $\mathrm{E}=$ ethyl acetate extract.

Table 2: Disc diffusion and liquid dilution MIC values for the $R$. canina extracts against P. mirabilis, P. vulgaris, K. pneumoniae, A. baylyi, P. aeruginosa and S. pyogenes growth $(\mu \mathrm{g} / \mathrm{mL})$.

\begin{tabular}{|c|c|c|c|c|c|c|c|c|c|c|c|c|}
\hline \multirow{2}{*}{ EXRACT } & \multicolumn{2}{|c|}{$\begin{array}{l}\text { P. mirabilis } \\
\text { (ATCC33304) }\end{array}$} & \multicolumn{2}{|c|}{$\begin{array}{c}\text { P. vulgaris } \\
\text { (ATCC21719) }\end{array}$} & \multicolumn{2}{|c|}{$\begin{array}{l}\text { K. pneumoniae } \\
\text { (ATCC31488) }\end{array}$} & \multicolumn{2}{|c|}{$\begin{array}{c}\text { A. baylyi } \\
\text { (ATCC21721) }\end{array}$} & \multicolumn{2}{|c|}{$\begin{array}{l}\text { P. aeruginosa } \\
\text { (ATCC39324) }\end{array}$} & \multicolumn{2}{|c|}{ S. pyogenes } \\
\hline & DD MIC & LD MIC & DD MIC & LD MIC & DD MIC & LD MIC & DD MIC & LD MIC & DD MIC & LD MIC & DD MIC & LD MIC \\
\hline W & 700 & 525 & 1080 & 868 & ND & - & ND & $>5000$ & ND & $>5000$ & $>5000$ & 3900 \\
\hline E & 200 & 185 & 586 & 365 & ND & - & ND & - & ND & - & ND & - \\
\hline $\mathbf{H}$ & ND & - & ND & - & 164 & 82 & ND & - & ND & - & ND & - \\
\hline \multicolumn{13}{|l|}{ Positive controls } \\
\hline Penicillin & ND & 2.5 & ND & 1.25 & ND & - & ND & - & ND & - & ND & - \\
\hline Chloramphenicol & ND & - & ND & - & ND & 1.25 & ND & 2.5 & ND & - & ND & - \\
\hline Tetracycline & ND & - & ND & 2.5 & ND & 1.25 & ND & 1.25 & ND & - & ND & 2.5 \\
\hline Negative control & ND & - & ND & - & ND & - & ND & - & ND & - & ND & - \\
\hline
\end{tabular}

$\mathrm{M}=$ methanol; $\mathrm{W}$ = water; $\mathrm{E}=$ ethyl acetate; $\mathrm{C}=$ chloroform; $\mathrm{H}=$ hexane. $\mathrm{DD}=$ disc diffusion; $\mathrm{LD}=$ liquid dilution. - indicates no inhibition at any dose tested. Numbers indicate the mean DD MIC and LD MIC values of triplicate determinations, expressed in $\mu \mathrm{g} / \mathrm{mL}$. ND = MIC could not be determined as only a single dose was tested.

as well as impetigo, streptococcal throat infections etc. It is noteworthy that this bacterial strain displayed substantial resaistance to most of the conventional antibiotics. Indeed, only gentamycin and tetracycline inhibited the growth of this bacterium although the relatively high MIC for tetracycline $(2.5 \mu \mathrm{g} / \mathrm{mL})$ indicates only low efficacy. Thus, it appears that this $S$. pyonenes strain is also resistant to tetracycline. Perhaps of greater interest, the combination of the methanolic extract and gentamycin was antagonistic. This combination should be avoided as a chemotherapeutic option to treat $S$. pyogenes infections. This was the only antagonistic result determined against $S$. pyogenes, indicating that all other combi- nations will not counter-indicate with the inhibitory properties of the conventional antibiotics.

\section{Varied ratio combination studies (isobolograms)}

\section{Synergistic interactions with chloramphenicol}

The combination of the methanolic R. canina extract and chloramphenicol induced synergistic interactions against K. pneumonia (Figure 7a) and A. baylyi (Figure $7 \mathrm{~b}$ ). These combinations were further examined using isobologram analysis across a range of extract:chloramphenicol 
Table 3: $\Sigma \mathrm{FIC}$ values of $R$. canina extracts in combination with conventional antibiotics against $P$. mirabilis, $P$. vulgaris, $K$. pneumoniae, A. baylyi, P. aeruginosa and S. pyogenes.

\begin{tabular}{|c|c|c|c|c|c|c|}
\hline & & Penicillin & Chloramphenicol & Gentamycin & Erythromycin & Tetracycline \\
\hline \multirow{5}{*}{ 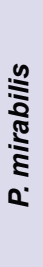 } & $M$ & 1.5 & - & 2 & - & - \\
\hline & W & - & - & 1.25 & - & - \\
\hline & $\mathrm{E}$ & 2.5 & - & 1.13 & - & - \\
\hline & C & - & - & - & - & - \\
\hline & $\mathrm{H}$ & - & - & - & - & - \\
\hline \multirow{5}{*}{$\begin{array}{l}\frac{n}{5} \\
\frac{\pi}{5} \\
\vdots \\
0\end{array}$} & M & 1.2 & - & 2.6 & - & 0.37 \\
\hline & W & 2.2 & - & 3.4 & - & 0.57 \\
\hline & $\mathrm{E}$ & 1.4 & - & 2.9 & - & 0.94 \\
\hline & C & - & - & - & - & - \\
\hline & $\mathrm{H}$ & - & - & - & - & - \\
\hline \multirow{5}{*}{ 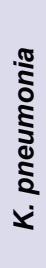 } & M & - & 0.46 & 2.7 & - & 0.29 \\
\hline & W & - & - & - & - & - \\
\hline & $\mathrm{E}$ & - & - & - & - & - \\
\hline & $\mathrm{C}$ & - & - & - & - & - \\
\hline & $\mathrm{H}$ & - & 0.8 & 3.5 & - & 0.55 \\
\hline \multirow{5}{*}{ 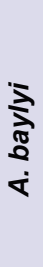 } & M & - & 0.5 & 3.2 & 1.4 & 0.6 \\
\hline & W & - & 2 & 3.5 & 1.8 & 0.8 \\
\hline & $\mathrm{E}$ & - & - & - & - & - \\
\hline & C & - & - & - & - & - \\
\hline & $\mathrm{H}$ & - & - & - & - & - \\
\hline \multirow{5}{*}{ 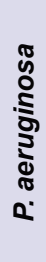 } & $M$ & - & - & 1.6 & - & - \\
\hline & W & - & - & 2.4 & - & - \\
\hline & $\mathrm{E}$ & - & - & - & - & - \\
\hline & C & - & - & - & - & - \\
\hline & $\mathrm{H}$ & - & - & - & - & - \\
\hline \multirow{5}{*}{ 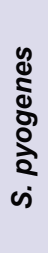 } & $M$ & - & - & $\underline{4}$ & - & 0.8 \\
\hline & w & - & - & 2.8 & - & 1.1 \\
\hline & $E$ & - & - & - & - & - \\
\hline & $\mathrm{C}$ & - & - & - & - & - \\
\hline & $\mathrm{H}$ & - & - & - & - & - \\
\hline
\end{tabular}

$\mathrm{M}=$ methanol; $\mathrm{W}=$ water; $\mathrm{E}=$ ethyl acetate; $\mathrm{C}=$ chloroform; $\mathrm{H}=$ hexane - indicates that the $\Sigma \mathrm{FIC}$ could not be determined. $\mathrm{M}=$ methanol; $\mathrm{W}$ = water; $\mathrm{E}=$ ethyl acetate; $\mathrm{C}=$ chloroform; $\mathrm{H}=$ hexane; - indicates that the $\Sigma \mathrm{FIC}$ could not be determined; Synergy (blue/bold highlighting) $=\leq 0.5$; Additive (green/italics highlighting) $=>0.5-1.0$; Indifferent (no highlighting) $=>$ $1.0-\leq 4$; Antagonistic (red/underlined highlighting) $=>4.0$. Numbers indicate the mean $\Sigma$ FIC values of 4 determinations.

ratios to identify the ideal ratios to obtain synergy. All combination ratios containing $20-60 \%$ of the methanolic extract produced synergistic interactions against K. pneumonia (Figure 7a). Thus, these combination ratios would be beneficial to enhance $K$. pneumonia growth inhibition. However, bacteria would be less likely to develop resistance when combinations are used in ratios which minimise the amount of conventional antibiotic used. Thus, for long term prophylactic treatment (as would be required to prevent and treat ankylosing spondylitis), the ideal extract:chloramphenicol ratio may be 60:40. However, when used for the treatment of acute infections (e.g. lung infections), the ratio which maximises the efficacy of the treatment (i.e. the 20:80 ratio) may be the preferred option.

Similarly, the methanolic $R$. canina extract in combination with chloramphenicol produced synergy against $A$. baylyi across a wide range of ratios (Figure $7 \mathrm{~b}$ ). Only the ratios containing between $10-20 \%$ methanolic $R$. canina extract, or the combination containing $90 \%$ extract, produced additive effects. All other combination ratios were synergistic. However, whilst combinations containing $50-80 \%$ extract are classified as 
Wang, et al:: Antibiotic Potentiation by Rosehip Extracts

Table 4: $\mathrm{LC}_{50}$ values determined for $R$. canina extracts in the Artemia nauplii and HDF bioassays following 24 hours exposure.

\begin{tabular}{ccc}
\hline \multirow{2}{*}{ Extract } & \multicolumn{2}{c}{$\mathrm{LC}_{50}$ value $(\mu \mathrm{g} / \mathrm{mL})$} \\
\cline { 2 - 3 } & ALA & HDF assay \\
\hline M & 1280 & - \\
W & 1850 & - \\
E & - & - \\
C & - & \\
H & - & - \\
PC & 48 & \\
\hline
\end{tabular}

- indicates that less than $50 \%$ mortality was induced by the extract at all concentrations tested. $100 \%$ mortality was induced by potassium dichromate (positive control) and none by the sea water (negative control) at $1000 \mu \mathrm{g} / \mathrm{mL}$. $\mathrm{ALA}=$ Atremia nauplii toxicity assay; $\mathrm{HDF}=$ human dermal fibroblast toxicity assay; $\mathrm{M}=$ methanolic extract; $\mathrm{W}=$ aqueous extract; $\mathrm{E}=$ ethyl acetate extract; $\mathrm{C}=$ chloroform extract; $\mathrm{H}=$ hexane extract.

synergistic interactions, they were on the cut off between synergy and additive interactions. Therefore, the combination containing $40 \%$ methanolic $R$. canina extract and $60 \%$ chloramphenicol was deemed to be the best combination ratio for prophylactic treatment to prevent multiple sclerosis, as well as decreasing the possibility of further increasing bacterial resistance to chloramphenicol.

\section{Synergistic interactions with tetracycline}

Combinations of the methanolic R. canina extract and tetracycline induced synergistic interactions against $P$. vulgaris (Figure 8a) and K. pneumonia (Figure $8 \mathrm{~b}$ ). All combination containing $30-70 \%$ of the extract were synergistic against both of these bacteria. Therefore, these ratios would be beneficial as treatment options to enhance the growth inhibitory activity against $P$. vulgaris and K. pneumonia. However, the $70 \%$ extract and 30\% tetracycline combination may be the ideal ratio for long prophylactic treatment, whilst the combination containing 30\% extract may be preferential for the treatment of acute infections.

\section{Quantification of toxicity}

No $\mathrm{LC}_{50}$ values were determined for the ethyl acetate, chloroform or hexane extracts as $<50 \%$ mortality was seen in all tested concentrations (Table 4). In contrast, $\mathrm{LC}_{50}$ values of 1280 and $1850 \mu \mathrm{g} / \mathrm{ml}$ were determined for the methanolic and aqueous extracts respectively. As extracts with $\mathrm{LC}_{50}$ values $<1000 \mu \mathrm{g} / \mathrm{ml}$ towards Artemia nauplii have previously been defined as being toxic in this assay, ${ }^{16}$ all extracts were deemed to be nontoxic. Furthermore, all plant extracts demonstrated a lack of toxicity towards normal human primary dermal fibroblasts, with cellular viability for all tests substantially $>50 \%$ of the untreated control. All extracts were therefore deemed to be either nontoxic.

\section{DISCUSSION}

This study investigated the ability of $R$. canina extracts to inhibit the growth of some bacterial triggers of autoimmune inflammatory diseases, both alone and in combination with conventional antibiotics. Several $R$. canina extracts were identified as effective bacterial growth inhibitors. The methanolic and aqueous extracts were particularly strong inhibitors of P. mirabilis, P. vulgaris and K. pneumoniae growth, with MIC values as low as $150 \mu \mathrm{g} / \mathrm{mL}$. Whilst these extracts also inhibited the growth of $A$. baylyi, P. aeruginosa and S. pyogenes, the MIC values were generally substantially $>1000 \mu \mathrm{g} / \mathrm{mL}$ and are thus indicative of only low to moderate inhibitory activity. Whilst a detailed investigation of the phytochemistry of the R. canina extracts was beyond the scope of this study, the qualitative phytochemical studies highlighted several phytochemical classes that may contribute to the bacterial growth inhibitory activity. Interestingly, the methanolic and aqueous $R$. canina extracts had relatively high abundances in polyphenolics, flavonoids, tannins, triterpenoids and saponins. Many studies have reported potent antibacterial activities for a wide variety of flavonoids. ${ }^{11}$ This has been attributed to a variety of mechanisms, including their ability to complex with extracellular and soluble proteins, as well as bacterial cell walls. ${ }^{18}$ Similarly, multiple tannins have broad spectrum antibacterial activity via a variety of intra- and extra-cellular mechanisms, including the precipitation of microbial proteins. ${ }^{19}$ Triterpenoids also have antibacterial activity, although the inhibitory mechanism is yet to be identified. ${ }^{20}$ It is likely that other phytochemical classes may also contribute to the growth inhibitory properties of these extracts. Therefore, phytochemical evaluation studies and bioactivity driven isolation of the active components are required to evaluate the mechanism of the $R$. canina extracts growth inhibitory activity.

The studies combining the extracts with conventional antibiotics were of perhaps greater interest. Several combinations displayed substantially greater potential as therapeutic agents against bacterial triggers of rheumatoid arthritis, ankylosing spondylitis and multiple sclerosis than the extracts or antibiotics alone. Four synergistic combinations were identified in this study, with two synergistic combinations each noted in combinations of the methanolic $R$. canina extract in combination with either chloramphenicol or tetracycline. The implications of a synergistic interaction include enhanced efficacy, thereby allowing lower dose administration, with reduced side effects and possibly reduced antimicrobial resistance, or conversely greater efficacy with administration of the same dosage..$^{15}$ Notably, the bacteria against which synergistic combinations were detected ( $P$. vulgaris, K. pneumonia and A. baylyi) were initially resistant to chloramphenicol and tetracycline. Thus, this study identified combinations of plant extracts and antibiotics which may repurpose relatively ineffective antibiotics and greatly enhance their efficacy, even against otherwise resistant bacterial strains. All of the extracts in synergistic combinations were high polarity extracts (methanol or water) suggesting the presence of a common active compound or class of compounds that may be responsible for the synergistic effects. Future studies are required to elucidate the potentiating mechanism(s) for the synergistic and additive combinations detected in our study.

Microbes have developed numerous resistance mechanisms to avoid the effects of antibiotics. One main method is through the use of multi-drug resistant (MDR) efflux pumps which are encoded chromosomally and are used to rapidly remove antibiotics that have entered the bacterial cells, thus rendering them resistant to the effects of the antibiotic. ${ }^{20,21}$ A single pump may allow the bacteria to escape several types of antimicrobials. When these efflux pumps are inhibited, the intracellular concentration of antibiotic will increase, allowing the treatment to once again be effective. Interestingly, many plants possess MDR pump inhibitors in order to enhance the activity of their own natural antimicrobial compounds. Such MDR pump inhibitors become effective tools when used in combination with some previously ineffective/resistance prone antibiotic compounds and several examples have previously been reported. ${ }^{22}$ Isoflavones isolated from Lupinus argenteus potentiate the activity of the natural plant antibiotic berberine as well as the synthetic fluoroquinoline antibiotic, norfloxacin as inhibitors of $S$. aureus growth. ${ }^{22}$ That study reported that the isoflavone allows a greater concentration of berberine to occur inside the bacteria by inhibiting the efflux mechanism (MDR pump). Similarly, Mezoneuron benthamianum and Securinega virosa extracts act as efflux pump inhibitors for fluoroquinolone, tetracycline and erythromycin in resistant strains of $S$. aureus (MRSA). ${ }^{23}$ As a consequence, the M. benthamianum ethanol extract and chloroform extract of $S$. virosa reduce 
the MIC (minimum inhibitory concentration) of norfloxacin against $S$. aureus by a factor of 4 .

In our study, all bacterial species were resistant to penicillin-G, chloramphenicol, erythromycin and tetracycline, with only low susceptibility or complete resistance to each antibiotic. All of these antibiotics are susceptible to resistance due to efflux pumps. ${ }^{22-28}$ A single pump can provide bacteria with resistance to a wide array of chemically and structurally diverse antibiotics and it is not uncommon for an organism to code for more than one efflux pump..$^{22,28}$ It is therefore imperative to identify agents that can block the efflux mechanism (efflux pump inhibitors - EPIs) or alter the process of efflux and in so doing, extend the life of existing antibacterial drugs. Plants produce various secondary metabolites that are used as defense mechanisms against pathogenic invaders. Some plants produce antimicrobials which, along with other compounds, inhibit the efflux of those antimicrobials from a bacterial cell. There are currently no EPI/antimicrobial drug combinations on the market, although research into identifying potential EPIs is ongoing. ${ }^{22}$ The synergistic interactions in our study suggests the possibility of a common EPI in the $R$. canina methanolic and aqueous extracts that could be inhibiting a MDR efflux pump in these bacteria.

Alternatively (or in addition to MDR efflux pumps), the bacteria screened in our study may have acquired genes encoding for reducedaffinity penicillin-binding protein $2 \mathrm{a}$ (PBP2a) (rendering $\beta$-lactam antibiotics ineffective) ${ }^{29}$ It is likely that as penicillin binding proteins are a group of protein enzymes, these phytochemicals may form nonspecific interaction and affect the bacterial cell wall biosynthesis. The $R$. canina extracts may also contain a $\beta$-lactamase inhibitor. $\beta$-lactamases are the major defense of gram-negative bacteria against $\beta$-lactam antibiotics. ${ }^{30}$ Clavulanic acid is an irreversible $\beta$-lactamase inhibitor, which in combination with $\beta$-lactam antibiotics can block the bacterial antimicrobial resistance mechanism. ${ }^{31}$ Further studies are required to identify whether extract compounds mirror the chemical and biological characteristics of clavulanic acid (i.e. the presence of a $\beta$-lactam ring).

Ultimately, the preparation of combinations of $R$. canina extracts (or purified compounds) with conventional antibiotic will depend on the nature of the pathogen and of the disease treated. In general, combinations of antibiotic with pure $R$. canina derived compounds would be preferred for acute infections as they are much less complex, easier to standardize and have lower chances of unwanted side effects. The use of crude extracts in these preparations is also effective and may still be acceptable to treat some diseases. However, when treating chronic illness, or using a combinational approach to prevent illness (as would be required in preventing autoimmune inflammatory diseases), the use of a pure potentiator compound in combination with the antibiotic may not be preferred. Continuous exposure of bacteria to a pure antibiotic (or to a combination of a single antibiotic and single potentiator) is likely to induce resistance to one or both of the compounds in the bacteria. Indeed, some E. coli strains are now resistant to amoxicillin-clavulanic acid combinations. ${ }^{32}$ However, crude plant extracts often contain numerous antibacterial compounds which may affect multiple bacterial targets. Thus, using a plant extract (rather than pure plant compounds) in combination with an antibiotic is less likely to result in resistant bacteria. Indeed, we were unable to find reports of any bacteria developing resistance to a crude plant extract. For this reason, when recommending preferred combination ratios throughout this study, we have recommended two different ratios for acute and chronic conditions. The lowest extract:highest antibiotic ratio which produced synergy has been deemed as the ideal ratio for treating acute bacterial infections, whilst we deemed the highest extract:lowest antibiotic ratio which produced synergy to be preferred for preventing and treating chronic disease

A further trend was evident in our study: most of the extract-antibiotic combinations which did not produce synergistic effects, generally did not greatly affect the efficacy of the antibiotic i.e. they appear to not counter-indicate with the antibiotics tested in this study. This is important as many users of herbal and traditional medicines self-diagnose/treat, often with multiple therapies concurrently. Thus, an understanding of drug herbal medicine interactions is important. Only a single combination tested in this study produced antagonistic interactions with the conventional antibiotics (against $S$. pyogenes in conjunction with gentamycin). This is an important finding and highlights that this combination should be avoided when treating $S$. pyogenes infections. Interestingly, previous studies indicate that antagonistic combinations of plant extracts with gentamycin are not uncommon. ${ }^{33}$

\section{CONCLUSION}

The results of this study demonstrate the potential of the $R$. canina extracts in inhibiting the growth of some bacterial triggers of autoimmune inflammatory diseases. The aqueous and methanolic extracts were good inhibitors of several microbes. However, the therapeutic potential of the $R$. canina extracts was far more apparent when tested in combination with conventional antibiotics as potentiators. Although the potentiation mechanisms are still unclear, compounds within $R$. canina extracts may mimic the actions of resistance modifying agents, thus potentiating the activity of several antibiotics that are relatively ineffective alone. Therefore, a combinational approach not only increases the effectiveness of drugs, but may also potentially reduce the side effects and reduce the development of drug resistant pathogens.

\section{ACKNOWLEDGEMENT}

Financial support for this work was provided by the Environmental Futures Research Institute and the School of Natural Sciences, Griffith University, Australia.

\section{ABBREVIATIONS}

DMSO: Dimethyl sulfoxide; $\mathbf{L C}_{50}$ : The concentration required to achieve 50\% mortality; MIC: Minimum inhibitory concentration.

\section{CONFLICT OF INTEREST}

The authors declare that they have no conflict of interests.

\section{REFERENCES}

1. Davies J, Davies D. Origins and evolution of antibiotic resistance. Microbiology and Molecular Biology Reviews. 2010;74(3):417-33.

2. Davies J. Where have all the antibiotics gone?. Canadian Journal of Infectious Diseases and Medical Microbiology. 2006;17(5):287-90.

3. WHO. The Evolving Threat of Antimicrobial Resistance: Options for Action. World Health Organization. 2014. http://apps.who.int/iris/bitstre am/10665/44812/1/9789241503181_eng.pdf.

4. Cheesman MJ, Ilanko A, Blonk B, Cock IE. Developing new antimicrobial therapies: Are synergistic combinations of plant extracts/compounds with conventional antibiotics the solution?. Pharmacognosy Reviews. 2017;11(22):57-72. DOI: 10.4103/phrev.phrev_21_17

5. Cock IE, Cheesman M. Chapter 23. The potential of plants of the genus Syzygium (Myrtaceae) for the prevention and treatment of arthritic and autoimmune diseases. In Bioaxctive Food as Dietary Interventions for Arthritis and Related Inflammatory Diseases, $2^{\text {nd }}$ edition; Elsevier Academic Press. 2019;401-24. https://doi.org/10.1016/B978-0-12-813820-5.00023-4

6. Vuuren SV, Viljoen A. Plant-based antimicrobial studies-methods and approaches to study the interaction between natural products. Planta Medica. 2011;77(11):1168-82

7. Cottarel G, Wierzbowski J. Combination drugs, an emerging option for antibacterial therapy. Trends in Biotechnology. 2007;25(12):547-55

8. Hemaiswarya S, Kruthiventi AK, Doble M. Synergism between natural products and antibiotics against infectious diseases. Phytomedicine. 2008;15(8):639-52.

9. Ziegler SJ. Fast and selective assay of I-ascorbic acid in rose hips by RP-HPLC coupled with electrochemical and/or spectrophotometric detection. Planta Medica 1986;52(5):383-7. 
Wang, et al:: Antibiotic Potentiation by Rosehip Extracts

10. Horváth, G, Molnár, P, Radó-Turcsi, E, et al. Carotenoid composition and in vitro pharmacological activity of rose hips. Acta Biochimica Polonica. 2012;59(1):12932.

11. Chrubasik C, Roufogalis BD, Müller-Ladner $U$, et al. A systematic review on the Rosa canina effect and efficacy profiles. Phytotherapy Research. 2008;22(6):725-33

12. Courtney J, Sirdaarta J, Matthews B, Cock IE. Tannin components and inhibitory activity of Kakadu plum leaf extracts against microbial triggers of autoimmune inflammatory diseases. Pharmacognosy Journal. 2015;7(1):18-31. DOI: 10.5530/pj.2015.7.2

13. Vesoul J, Cock IE. The potential of bunya nut as an antibacterial food agent Pharmacognosy Communications. 2012;2(1):72-9. DOI: 10.5530/pc.2012.1.13

14. Eloff JN. A Sensitive and quick microplate method to determine the minimal inhibitory concentration of plant extracts for bacteria. Planta Medica. 1998;64(08):711-3.

15. Hübsch Z, ZyI VRL, Cock IE, et al. Interactive antimicrobial and toxicity profiles of conventional antimicrobials with Southern African medicinal plants. South African Journal of Botany. 2014;93:185-97. DOI: 10.1016/j.sajb.2014.04.005

16. Ruebhart D, Wickramasinghe W, Cock IE. Protective efficacy of the antioxidants vitamin E and Trolox against Microcystis aeruginosa and microcystin-LR in Artemia franciscana nauplii. Journal of Toxicology and Environmental Health, Part A 2009;72(24):1567-75. DOI: 10.1080/15287390903232459

17. Shalom J, Cock IE. Terminalia ferdinandiana Exell. Fruit and leaf extracts inhibit proliferation and induce apoptosis in selected human cancer cell lines. Nutrition and Cancer. 2018;70(4):1-15. DOI: 10.1080/01635581.2018.1460680

18. Kaur GJ, Arora DS. Antibacterial and phytochemical screening of Anethum graveolens, Foeniculum vulgare and Trachyspermum ammi. BMC Complementary and Alternative Medicine. 2009;9(1):30.

19. Buzzini P, Arapitsas P, Goretti M, et al. Antimicrobial activity of hydrolysable tannins. Medicinal Chemistry. 2008;8(2):1179-87.

20. Chung PY, Navaratnam P, Chung LY. Synergistic antimicrobial activity between pentacyclic triterpenoids and antibiotics against Staphylococcus aureus strains. Annals of Clinical Microbiology and Antimicrobials. 2011;10(1):25

21. Cowan MM. Plant products as antimicrobial agents. Clinical Microbiology Reviews. 1999;12(4):564-82.

22. Morel C, Stermitz FR, Tegos G, Lewis K. Isoflavones as potentiators of antibacterial activity. Journal of Agriculture and Food Chemistry. 2003;51(19):5677-9.
23. Dickson RA, Houghton PJ, Hylands PJ, Gibbons S. Antimicrobial, resistancemodifying effects, antioxidant and free radical scavenging activities of Mezoneuron benthamianum Baill., Securinega virosa Roxb. and WIId. and Microglossa pyrifolia Lam. Phytotherapy Research. 2006;20(1):41-5.

24. Abreu AC, McBain AJ, Simoes M. Plants as sources of new antimicrobials and resistance-modifying agents. Natural Product Reports. 2012;29(9):1007-21.

25. Stavrim M, Piddock LJV, Gibbons S. Bacterial efflux pump inhibitors from natural sources. Journal of Antimicrobial Chemotherapy. 2007;59(6):1247-60.

26. Santiago C, Pang EL, Lim KH, Loh HS, Ting KN. Inhibition of penicillin-binding protein 2a (PBP2a) in methicillin resistant Staphylococcus aureus (MRSA) by combination of ampicillin and a bioactive fraction from Duabanga grandiflora. BMC Complementary and Alternative Medicine. 2015;15(1):178.

27. Yap PSX, Yiap BC, Ping HC, Lim SHE. Essential oils, a new horizon in combating bacterial antibiotic resistance. The Open Microbiology Journal. 2014;8:6.

28. Sahli AA, Abdulkhair WM. Inhibition of beta-lacamase enzyme of Pseudomonas aeruginosa by clavulanic acid of Rumex vesicarius L. African Journal of Agricultutal Research. 2011;6(12):2908-15.

29. Dickson RA, Houghton PJ, Hylands PJ, Gibbons S. Antimicrobial, resistancemodifying effects, antioxidant and free radical scavenging activities of Mezoneuron benthamianum Baill., Securinega virosa Roxb. and Wlld. and Microglossa pyrifolia Lam. Phytotherapy Research. 2006;20(1):41-5.

30. Stavrim M, Piddock LJV, Gibbons S. Bacterial efflux pump inhibitors from natural sources. Journal of Antimicrobial Chemotherapy. 2007;59(6):1247-60.

31. Ramón-Garcia S, DelGonzález RR, Villarejo AS, Sweet GD, Cunningham F, Bar ros $D$, et al. Repurposing clinically approved cephalosporins for tuberculous therapy. Scientific Reports. 2016;6:34239.

32. Oteo J, Campos J, Lázaro E, Cuevas O, Garcia-Cobos S, Pérez-Vázquez M, et al. Spanish Members of EARSS. Increased amoxicillin-clavulanic acid resis tance in Escherichia coli blood isolates, Spain. Emerging Infectious Diseases. 2008;14(8):1259-62

33. Toroglu S. In-vitro antimicrobial activity and synergistic/antagonistic effect of interactions between antibiotics and some spice essential oils. Journal of Environmental Biology. 2011;32(1):23-9.

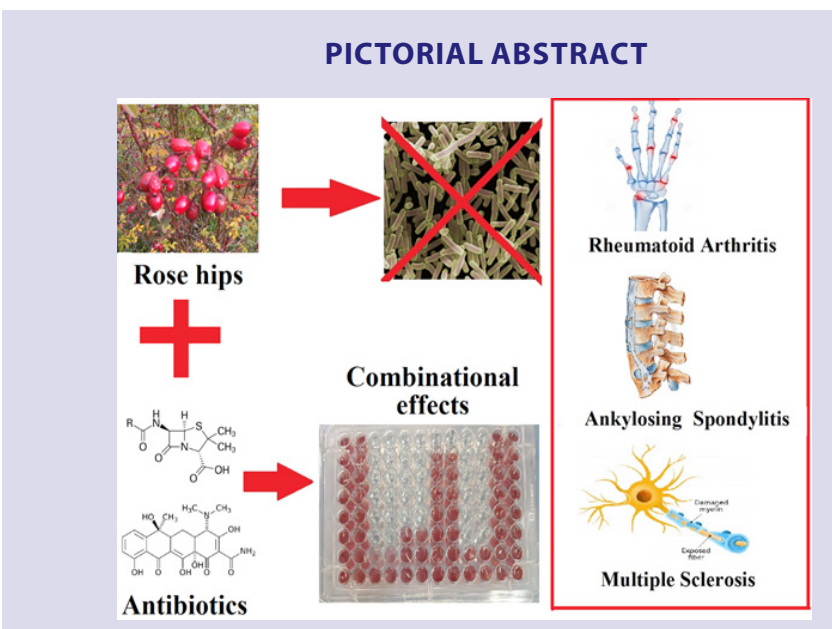

\section{SUMMARY}

- $\quad$ R. canina rose hip extracts were screened for the ability to block the growth of a panel of bacterial triggers of autoimmune inflammatory diseases.

- The antibacterial activity was quantified by determining the MIC values of each extract.

- $\quad$ The extracts were also tested in combination with conventional antibiotics and the class of interaction was determined

- $\quad$ Synergistic combinations were screened at various ratios to determine the ideal ratios to provide synergy.

- $\quad$ Toxicity of the $B$. acerifolius leaf and flower extracts was determined using the Artemia nauplii and HDF cell viability toxicity bioassays.

\section{ABOUT AUTHORS}

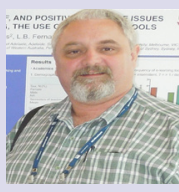

Dr. Ian Cock leads a research team in the Environmental Futures Research Institute and the School of Natural Sciences at Griffith University, Australia. His research involves bioactivity and phytochemical studies into a variety of plant species of both Australian and international origin, including Aloe vera, South Asian and South American tropical fruits, as well as Australia plants including Scaevola spinescens, Pittosporum phylliraeoides, Terminalia ferdinandiana (Kakadu plum), Australian Acacias, Syzygiums, Petalostigmas and Xanthorrhoea johnsonii (grass trees). This range of projects has resulted in nearly 200 publications in a variety of peer reviewed journals. 